\title{
Directional Track Selection Technique in CR39 SSNTD for low- yield reaction experiments
}

\author{
Francesco Ingenito ${ }^{1}$, Pierluigi Andreoli ${ }^{1}$, Dimitri Batani ${ }^{2}$, Aldo Bonasera ${ }^{3}$, Guillaume Boutoux ${ }^{2}$, Frederic Burgy $^{2}$, \\ Mattia Cipriani ${ }^{1}$, Fabrizio Consoli ${ }^{1}$, Giuseppe Cristofari ${ }^{1}$, Riccardo De Angelis $^{1 *}$, Giorgio Di Giorgio ${ }^{1}$, \\ Jean Eric Ducret ${ }^{2}$, Danilo Giulietti ${ }^{4}$, and Katarzyna Jakubowska ${ }^{2,5}$ \\ ${ }^{1}$ ENEA, Fusion and Technologies for Nuclear Safety Department, C. R. Frascati, Via E. Fermi 45, 00044 Frascati (Roma), Italy \\ ${ }^{2}$ Centre d'Etudes Lasers Intenses et Applications (CELIA), Université de Bordeaux, CNRS, CEA, 351 cours de la liberation \\ 33405 Talence, France \\ ${ }^{3}$ Cyclotron Institute, Texas AM University (TAMU), 120 Spence St, College Station, TX 77840, United States \\ ${ }^{4}$ Physics Department of the University and INFN, Largo B. Pontecorvo 3, 57127, Pisa, Italy \\ ${ }^{5}$ Institute of Plasma Physics and Laser Microfusion (IPPLM), Hery Street 23, 01-497 Warsaw, Poland, Poland
}

\begin{abstract}
There is a great interest in the study of $\mathrm{p}-{ }^{11} \mathrm{~B}$ aneutronic nuclear fusion reactions, both for energy production and for determination of fusion cross-sections at low energies. In this context we performed experiments at CELIA in which energetic protons, accelerated by the laser ECLIPSE, were directed toward a solid Boron target. Because of the small cross-sections at these energies the number of expected reactions is low. CR39 Solid-State Nuclear Track Detectors (SSNTD) were used to detect the alpha particles produced. Because of the low expected yield, it is difficult to discriminate the tracks due to true fusion products from those due to natural background in the CR39.To this purpose we developed a methodology of particle recognition according to their direction with respect to the detector normal, able to determine the position of their source. We applied this to the specific experiment geometry, so to select from all the tracks those due to particles coming from the region of interaction between accelerated protons and solid boron target. This technique can be of great help on the analysis of SSNTD in experiments with low yield reactions, but can be also generally applied to any experiment where particles reach the track detector with known directions, and for example to improve the detection limit of particle spectrometers using CR39.
\end{abstract}

\section{Introduction}

Solid-State Nuclear Track Detectors (SSNTD) are samples of solid materials where exposition to ionizing radiation generates tracks due to local damaging of the detector [1]. In polymeric materials, such as CR39, tracks are caused by the breaking of the long polymer chains. Along these tracks the material is more susceptible to chemical attack. Therefore, these regions have much faster velocity of etching with respect to undamaged material. Etching of several hours allows for enlarging of the damage to micrometer dimensions, which can be observed with a microscope [1]. Information on particle energy can be determined by track dimension and etchrate of track compared to that of the bulk. Moreover, circular pits are generated if the particles enter the surface at normal incidence, otherwise elliptical pits are generated, where ellipticity and orientation of the pit mouth are related to the direction of incidence.

These detectors are widely used in different fields and are fundamental in environments heavily affected by electromagnetic radiation of high intensity, where active electronic detectors will be not applicable [2-4]. In particular, they are the key diagnostics in investigation of low-yield nuclear reactions, such as the neutron-less $\mathrm{p}-{ }^{11} \mathrm{~B}$ reactions generated by laser-matter interaction [5-12]. There is a strong interest in their application, both for energy production and for determining the reaction crosssections at low energies for astrophysical studies. One of the main problems in this context is the discrimination of a very small number of fusion products with respect to the background.

\section{Experiment description}

In this work we show results of experiments performed at CELIA laboratories in Bordeaux. An accelerated particle beam was produced by Target Normal Sheath Acceleration (TNSA) [13], focusing the ECLIPSE laser beam on $10 \mu \mathrm{m}$ spots on an aluminium thin foil of $6 \mu \mathrm{m}$ thickness [12]. The laser parameters were: $\lambda=800 \mathrm{~nm}$, up to $170 \mathrm{~mJ}$ energy in 35 fs of FWHM (Full Width Half Maximum), temporal contrast of $4 \cdot 10^{-9}$ at $12 \mathrm{~ns}$ and $10^{-5}$ at ASE (Amplified Spontaneous Emission) level, energy stability $1.5 \%$ RMS (Root Mean Square) shot to shot. The Intensity was $10^{18-19} \mathrm{Wcm}^{-2}$. As shown in Figure 1 and described in more details in reference [12], a proton beam

* Corresponding author: riccardo.deangelis@enea.it 
with energies up to $\sim 300 \mathrm{keV}$ energy was generated in this configuration and directed to a CR39 plate shielded with a thick Al foil. A hole on this plate, placed in correspondence of that on the thick Al, allowed protons to reach the solid $\mathrm{B}$ sample. The expected reaction is $\mathrm{p}+{ }^{11} \mathrm{~B}$ $\rightarrow 3 \alpha$ [5-12]. The side of the CR39 detector facing the B target was unfiltered and so directly exposed to the alpha particles, but also to the recoil protons from target, which represented a source of noise to the detector.

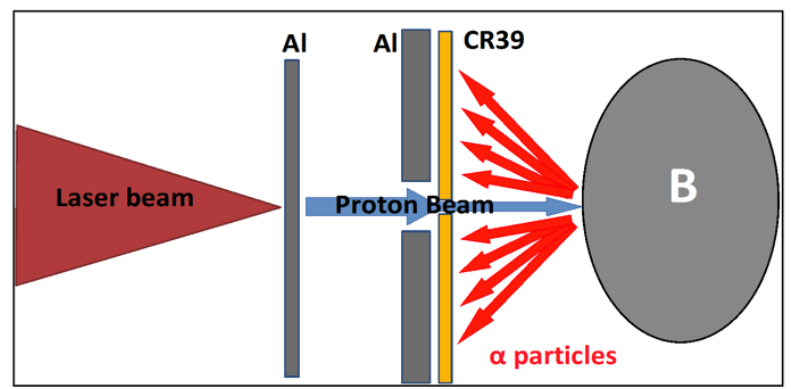

Fig. 1. Experimental set-up.

\subsection{CR39 SSNTD analysis}

The CR39 detectors exposed to the radiation were etched with a $\mathrm{NaOH}$ solution $6.25 \mathrm{M}$ at $70^{\circ} \mathrm{C}$ for 7 hours. Under these conditions, tracks with diameter of about $20 \mu \mathrm{m}$ are expected for alphas in the $2-4 \mathrm{MeV}$ range generated by possible $\mathrm{pB}$ fusions [5-12].

After the etching, the samples were scanned with an automated procedure by a reflection microscope, and this led to reconstruct a square image of $20 \mathrm{~mm}$ side. This image was analyzed with the ImageJ program [14] and its built-in particle-counting routine.

Both the images and the particle counter results showed 'noise', in the form of a high number of equivalent little tracks with a diameter of a few microns. In some cases these noise tracks also formed clusters, which were recognized as a single big track by the counter program. A careful recognition process is thus necessary, which would require a human operator performing it. Of course, this is a time-consuming and tedious work, which limits appreciably the extension of the detector surface that can be analyzed. This is a drawback in low-rate reactions as $\mathrm{pB}$. Moreover, a giga-pixel image was produced as results of microscope scanning. For all of these reasons a fully automatic and reliable selection method was needed.

\section{The method}

Track shape on SSNTDs is influenced by a number of parameters, some due to the etching conditions and others to the geometry of the particle collision [15]. In general, the pit mouth of the etched track can be described as an ellipse with the major axis along the particle propagation direction. We used this specific feature to discriminate tracks actually due to the reactions of our interest from those of background. In the scheme of Figure 1, possible alpha particles imping on the CR39 have a specific propagation direction on any given point of the detector. This Directional Track Selection (DTS) method was applied only to tracks having transversal dimensions compatible with those expected for alpha particles up to 4 $\mathrm{MeV}$, and this greatly improved the procedure.

\subsection{Determination of key parameters}

Here we explain the steps followed in the procedure.

\subsubsection{Determination of the particle direction}

As already mentioned, the shape of the track is approximately an ellipse. Taking advantage of the specific ImageJ built-in function, each track is fitted with the proper ellipse and its major axis direction is determined by the $\varphi$ angle with the horizontal axis of the image. From this information it is possible to infer the direction of the possible incident particle.

\subsubsection{Determination of the expected particle direction}

The expected particle direction is the actual direction of particles emitted by a given source. To find it, as a first step we projected the particle source on the detector plane, as indicated in Figure 2. The grey circle in that picture is the projection on the detector plane of the region of boron interacting with the proton beam, as shown in Figure 1. We set our reference system in the center of this circle. In this reference system the expected particle direction is represented by the segment of length d connecting the origin of the reference system to the center of the track image, and it is individuated by the $\theta$ angle it forms with the horizontal axis.

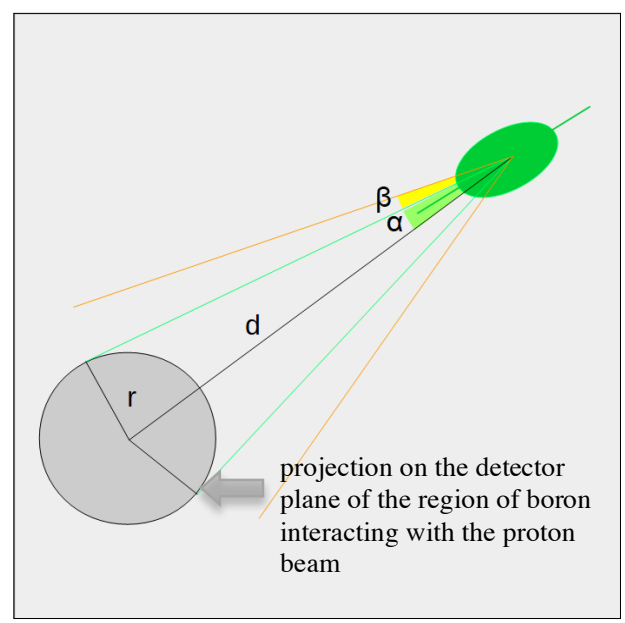

Fig. 2. Basic scheme for parameters of track selection.

\subsubsection{Determination of the acceptance angle}

Since the source has a specific extension, a particular track can be due to particles emitted by any point within source projection. Moreover, the uncertainties on the position, shape and extension of the source, on position of the track center and on the major-axis direction must be also taken into account. To this end, we define an overall 
acceptance angle, calculated as the sum of two contributions. As shown in Figure 2, the first term accounts for the possible provenience of particles from a generic point on the source:

$$
\alpha=\arcsin (\mathrm{r} / \mathrm{d})
$$

where $\mathrm{r}$ is the radius of the circle including the projection of the source zone on the detector plate. The second term $\beta$ is numerically evaluated for each image from the tolerances on the track ellipsoid drawn by the imaging program and the tolerances in assessing the source dimension and center position. The resulting $\gamma=\alpha+\beta$ acceptance angle is schematically indicated in Figure 2.
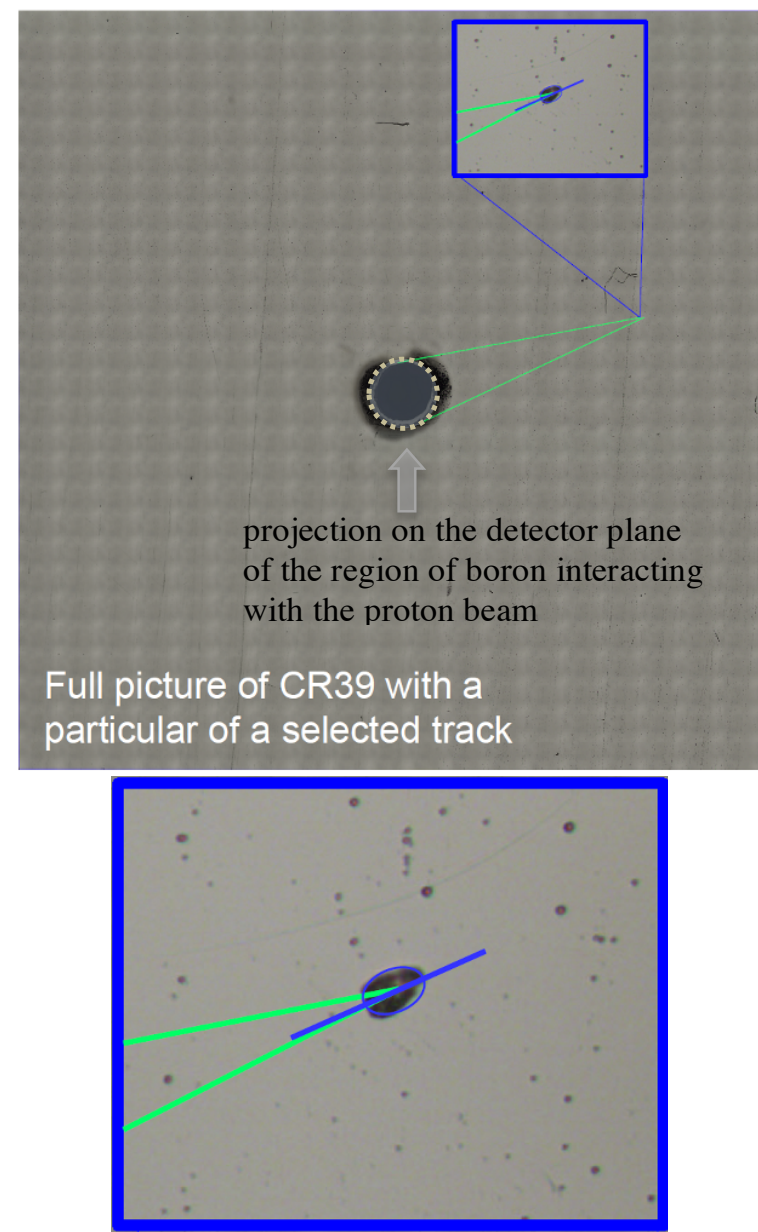

Fig. 3. In the top part of the picture there is the complete CR39 image obtained as an array of multiple microscope scans. A detail of one of these scans is shown, and it is also supplied in the lower part of the figure, enlarged with improved resolution (the size of the enlarged image is $180 * 225 \mu \mathrm{m}$ ). Particle direction, fitting ellipse and acceptance range are indicated.

\subsection{Track selection}

After the determination of the particle direction, the expected particle direction and the acceptance angle, the numerical process of selection can be performed. As previously mentioned, this is applied only to the tracks having dimensions compatible to alpha particles generated by $\mathrm{p}-\mathrm{B}$ fusion events. Among these, the tracks having $|\varphi-\theta| \leq \gamma$ are selected. In this way the operator has a reduced number of tracks to control for possible further validation.

In Figure 3 we show a picture obtained by the microscope scan of a CR39 exposed to a cumulative experiment of 300 nearly identical shots on fresh portions of the target, with average energy on Al target of $\sim 110 \mathrm{~mJ}$, and good shot stability. This is the appearance of a recollection of many scanned images in a big one. At the picture center we see the hole of the CR39, with an annular blur due to heavy radiation damage, caused by boundary effects due to particle passage through the hole, backscattering of particles impinging on the B surface and X-ray flux coming from bremsstrahlung of particles on B surface. One of the pictures composing this array is enlarged, and also shown with improved resolution in the lower part of the same figure. It is possible to observe a specific track selected by the numerical process, with the blue ellipse resulting from the fitting procedure, the associated particle direction indicated by a blue segment, and the $\gamma$ acceptance range represented by the two green lines.

As preliminary results of this technique we applied the selection procedure to all the detected traces. The first selection step, based only on the transversal dimension of the track, returned 112 cases compatible with emitted alphas from $\mathrm{pB}$ fusions. The application of the Directional Track Selection was able to reduce that number to only 12 traces, resulting in a more than 9 times decrease.

\section{Conclusions}

The great reduction of background indicated by the preliminary results of application of the presented selection technique allows for detailed individual analysis of the selected tracks by human operator with much lower effort. The technique is still in an early development stage, but allowed a far more efficient detector analysis. For this reason it can be of great importance when reactions with low yield (as in this $\mathrm{p}^{11} \mathrm{~B}$ case) are studied. Moreover, the application of this methodology can be very important to improve the background reduction in many different experiment types where it is possible to have geometrical constraints for direction of particles reaching the track detector. For this reason this methodology can be very powerful for improving the detection limit when CR39 are used in magnetostatic, electrostatic or Thomson particle spectrometers.

This work has been carried out within the framework of the EUROfusion Consortium and has received funding from the Euratom research and training programme 2014-2018 under grant agreement No 633053. The views and opinions expressed herein do not necessarily reflect those of the European Commission. The authors acknowledge the support of the COST Action MP1208 "Developing the Physics and the Scientific Community for Inertial Fusion".

\section{References}

1. S. A. Durrani, R. K. Bull, Solid State Nuclear Track Detection Principles, Methods and Applications, (Pergamon Press, 1987). 
2. J. Raimbourg, Rev. Sci. Instrum. 75, 4234 (2004).

3. C.G. Brown Jr., et al, J. Phys.: Conf. Ser. 244, 032001 (2010).

4. F. Consoli, et al., Phys. Procedia 62, 11 (2015).

5. V.S. Belyaev, et al, Phys. Rev. E 72, 026406 (2005).

6. A. Bonasera, A. Caruso et al, Proceedings of the 4th International Conference on Fission and Properties of Neutron Rich Nuclei, 11-17 November 2007, Sanibel Island, USA.

7. S. Kimura, A. Anzalone, A. Bonasera, Phys. Rev. E 79, 038401 (2009).

8. C. Labaune, et al, Nat. Comm. 4, 2506 (2013).

9. A. Picciotto, et al, Phys. Rev. X 4, 031030 (2014).

10. C. Baccou, et al, Laser Part. Beams 33, 117 (2015).

11. C. Baccou, et al, Rev. Sci. Instrum. 86, 083307 (2015).

12. D. Giulietti, et al, Nucl. Instrum. Meth. B 402, 317 (2017)

13. A. Macchi, et al, Rev. Mod. Phys. 85, 751 (2013).

14. Available at https://imagej.nih.gov/ij/

15. D. Nikezic, K.N. Yu, Mater. Sci. Eng. R-Rep. 46, 51 (2004). 